\title{
Comparison Between the Inside-Outside Algorithm and the Viterbi Algorithm for Stochastic Context-Free Grammars*
}

\author{
Joan-Andreu Sánchez, José-Miguel Benedí , Francisco Casacuberta \\ Departamento de Sistemas Informáticos y Computación \\ Universidad Politécnica de Valencia \\ Camino de Vera s/n, 46071 Valencia (Spain) \\ e-mail: \{jandreu,jbenedi,fcn\}@dsic.upv.es
}

\begin{abstract}
The most popular algorithms for the estimation of the probabilities of a context-free grammar are the Inside-Outside algorithm and the Viterbi algorithm, which are Maximum Likelihood approaches. The difference between the logarithm of the likelihood of a string and the logarithm of the likelihood of the most probable parse of a string is upper bounded linearly by the length of the string and the logarithm of the number of non-terminal symbols. However, this theoretical bound is too pessimistic. For this reason, an experimental work to show the behaviour of the two functions in practical cases is necessary.
\end{abstract}

\section{Introduction}

In Syntactic Pattern Recognition, Stochastic Context-Free Grammars (SCFG) are an adequate alternative to Stochastic Regular Grammars for representing syntactic-semantic constraints. Recently, some applications of SCFG have been proposed for Language Modeling [8, 5, 18], Acoustic-Phonetic Decoding [11], ADN Sequences Modeling [17], etc. One of the reasons for using these models is their ability to establish long-term statistical dependencies among primitives.

One problem related to SCFG is the learning of the rules and/or the probability distributions associated to the set of rules from training strings. Interesting Grammatical Inference techniques have been proposed elsewhere for learning the rules of the grammar [16]. In addition, several methods have been proposed for estimating rule probabilities in order to maximize the likelihood of a set of training strings (Inside-Outside algorithm -IO-) $[1,10,9,19,4]$; the likelihood of the best derivations of a set of training strings (for abbreviation we call this approximate likelihood) (Viterbi algorithm) [14, 4] or to improve the recognition accuracy on the training data [2].

The 10 algorithm and the Viterbi algorithm run in an iterative manner by modifying the probabilities of the rules until a local maximum [11] is achieved. In each iteration, both algorithms have a time complexity which is cubic with

\footnotetext{
* Work partially supported by the Spanish CICYT under contract TIC95/0884-C04.
} 
the longest string in the sample and linear with the number of training strings, linear with the number of non-terminal symbols and linear with the number of rules with the same non-terminal symbol in the left side. In the worst case, this last factor can be quadratic with the number of non-terminal symbols. However, the real computational cost of the Viterbi algorithm is significantly lower than the IO algorithm.

Taking the previous considerations into account, one aspect which would be interesting to study is the behaviour of the function that the IO algorithm maximizes when the Viterbi algorithm is used for estimating the probabilities of the rules. It would be equally important to know how the approximate likelihood evolves when the IO algorithm is used in the training phase. Some work has been done in this direction for Hidden Markov models $[12,13]$ which indicates that an upper bound can be established between the difference of the two functions for a given model.

In the context-free case, a similar theoretical bound can be established, but this bound may be very pessimistic (Section 2). In addition, an empirical study was carried out (Section 3) to show how the two functions evolve in practice depending on which algorithm is used in the training phase. This experimentation offers some significant conclusions (Section 4).

\section{On the Relation between the Inside-Outside and Viterbi Algorithms}

A Context-Free Grammar (CFG) $G$ is a four-tuple $(N, \Sigma, R, S)$, where $N$ is a finite set of non-terminal symbols, $\Sigma$ is a finite set of terminal symbols $(N \cap \Sigma=$ $\emptyset), R$ is a finite set of rules of the form $A \rightarrow \alpha\left(A \in N\right.$ and $\left.\alpha \in(N \cup \Sigma)^{*}\right)$ and $S$ is the initial symbol $(S \in N)$. A CFG in Chomsky Normal Form (CNF) is a CFG in which the rules are of the form $A \rightarrow B C$ or $A \rightarrow a(A, B, C \in N$ and $a \in \Sigma)$.

A SCFG $G_{s}$ is a pair $(G, q)$, where $G$ is a CFG and $\left.\left.q: R \rightarrow\right] 0,1\right]$ is a function such that:

$$
\forall A \in N, \quad \sum_{\alpha \in(N \cup \Sigma)^{*}} q(A \rightarrow \alpha)=1 .
$$

A left-deritavion of $x \in \Sigma^{*}$ in $G, D(x)$, is a sequence of rules $\left(r_{1}, \ldots, r_{m}\right)$ such that:

$$
D(x)=\left[S \stackrel{r_{7}}{\Rightarrow} \alpha_{1} \stackrel{r_{3}}{\Rightarrow} \alpha_{2} \stackrel{r_{3}}{\Rightarrow} \ldots \stackrel{r_{m}}{\Rightarrow} x\right],
$$

where $\alpha_{i} \in(N \cup \Sigma)^{*} 1 \leq i \leq m-1$, and $r_{i}$ rewrites the left-most non-terminal of $\alpha_{i-1}$.

If $D(x)$ is a left-derivation, a single parse tree, $T(x)$, can be associated with it [6]. The probability of a parse tree $T(x)$ is defined as the probability of the associated derivation $D(x)$, which in turn, is defined as:

$$
\operatorname{Pr}_{G}(x, T(x) \mid q)=\operatorname{Pr}_{G}(x, D(x) \mid q)=q\left(r_{1}\right) \cdot \ldots \cdot q\left(r_{m}\right) .
$$


The probability of generating $x \in \Sigma^{*}$ by $G_{s}$ is:

$$
\operatorname{Pr}_{G}(x \mid q)=\sum_{\forall T(x)} \operatorname{Pr}_{G}(x, T(x) \mid q) .
$$

The probability of the best parse tree can be defined as:

$$
\widehat{\operatorname{Pr}}_{G}(x \mid q)=\max _{\forall T(x)} \operatorname{Pr}_{G}(x, T(x) \mid q) .
$$

Given a sample from $\Sigma^{*}$, the function $q$ can be appropriately trained to locally maximize the likelihood of the sample (using the IO algorithm) or the likelihood of the most probable parse tree (Viterbi algorithm). Both algorithms have a time complexity $O\left(|x|^{3} \cdot|N| \cdot B r\right)$, where $B r$ is the maximum number of rules with the same non-terminals in the left side of the rule, $|N|$ is the number of non-terminal symbols and $|x|$ is the length of the longest string in the sample. In the worst case, $B r=|N|^{2}$; therefore, the time complexity can be cubic with the number of non-terminals.

We are interested in the theoretical study of the difference between (1) and (2). An upper bound on this difference can be derived in a way similar to the one in $[12,13]$.

Given a string $x \in \Sigma^{*}$, the first step is to obtain the maximum number of parse trees that can derive from $S$ to $x$ in $G_{s}$. Suppose that $G_{s}$ is in CNF. This means that any parse tree will be similar to the one in Fig. 1.

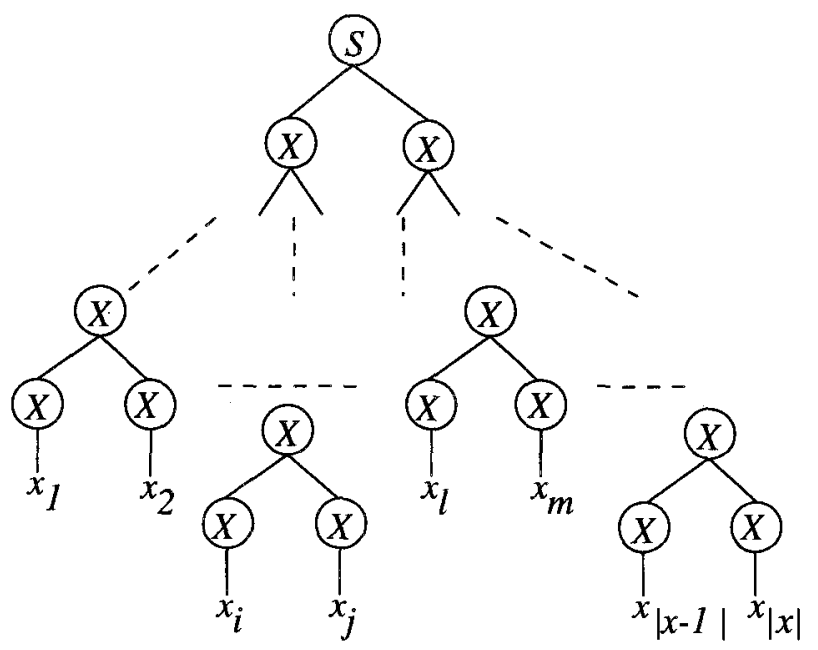

Fig. 1. General parse tree $T(x)$ for a string $x_{1} x_{2} \ldots x_{|x|} . X$ represents any symbol of $N . T^{\prime}(x)$ is obtained from $T(x)$ by removing nodes labeled with terminal symbols. In $T^{\prime}(x)$ all internal nodes have degree 2 . 
In this tree, the internal nodes are labeled with non-terminal symbols and the leaf nodes are labeled with terminal symbols. If the leaf nodes of that tree are removed, a binary tree, $T^{\prime}(x)$, is obtained where all internal nodes have degree 2 (internal degree). Taking the properties of a binary tree into account, the number of leaves of $T^{\prime}(x)$ will be $|x|$ (shadowed in Fig. 1) and the number of internal nodes will be $|x|-1$. Therefore, the total number of nodes will be $2|x|-1$. The maximum number of parse trees for a string $x$ in $G_{s}$ will be the number of different unlabeled binary trees (with internal degree 2) with $2|x|-1$ nodes times the number of possible combinations of labeling all nodes of $T^{\prime}(x)$ with non-terminal symbols.

Let $a_{m}$ be the number of unlabeled binary trees (with internal degree 2) with $m$ internal nodes $(m \geq 1)[7]$ :

$$
a_{m}=\sum_{i=0}^{m-1} a_{i} a_{m-i-1},
$$

with $a_{0}=1$. A non-recursive formula for this expression is [15]:

$$
a_{m}=\frac{1}{m+1} \frac{(2 m) !}{m ! m !} \leq \frac{1}{m+1} \frac{4^{m}}{\sqrt{\pi m}} .
$$

On the other hand, the number of all possible combinations of labeling all nodes of $T^{\prime}(x)$ with non terminal symbols is $|N|^{2|x|-2}$. The value $2|x|-2$ corresponds to the number of nodes in the tree minus the root node of the tree, which is always labeled with $S$. Hence, the maximum number of parse trees for a string $x$ in $G_{s}$ will be $a_{|x|-1} \cdot|N|^{2|x|-2}$ with $|x|>1$.

We now define the relation between (1) and (2) for a string $x$ in $G_{s}$ with $|x|>1$.

$$
\begin{aligned}
\ln \max _{\forall T(x)} \operatorname{Pr}_{G}(x, T(x) \mid q) & \leq \ln \sum_{\forall T(x)} \operatorname{Pr}_{G}(x, T(x) \mid q) \\
& \leq \ln \frac{1}{|x|} \frac{4^{|x|-1}}{\sqrt{\pi(|x|-1)}}|N|^{2|x|-2} \max _{\forall T(x)} \operatorname{Pr}_{G}(x, T(x) \mid q) \\
& \leq \ln \frac{2^{2|x|-2}}{|x| \sqrt{(|x|-1)}}|N|^{2|x|-2}+\ln \max _{\forall T(x)} \operatorname{Pr}_{G}(x, T(x) \mid q) \\
& \leq 2|x| \ln 2|N|-\ln |x| \sqrt{|x|-1}+\ln \max _{\forall T(x)} \operatorname{Pr}_{G}(x, T(x) \mid q) \\
& \leq 2|x| \ln 2|N|+\ln \max _{\forall T(x)} \operatorname{Pr}_{G}(x, T(x) \mid q) .
\end{aligned}
$$

Finally, we obtain the following result:

$$
\ln \operatorname{Pr}_{G}(x \mid q)-\ln \widehat{\operatorname{Pr}}_{G}(x \mid q) \leq 2|x| \ln 2|N| .
$$

This upper bound can be used to estimate the differences in the likelihood in the probabilistic estimation, if an efficient algorithm exists to achieve global maxima of the corresponding objective functions. 
We define for a string $x$ :

$$
\begin{aligned}
q^{*} & =\arg \max _{q} \operatorname{Pr}_{G}(x \mid q), \\
\hat{q} & =\arg \max _{q} \max _{\forall T(x)} \operatorname{Pr}_{G}(x, T(x) \mid q) .
\end{aligned}
$$

From these definitions we can derive:

$$
\begin{aligned}
\ln \max _{\forall T(x)} \operatorname{Pr}_{G}(x, T(x) \mid \hat{q}) & \leq \ln \operatorname{Pr}_{G}(x \mid \hat{q}) \leq \ln \operatorname{Pr}_{G}\left(x, q^{*}\right) \\
& \leq \ln \max _{\forall T(x)} \operatorname{Pr}_{G}\left(x, T(x) \mid q^{*}\right)+2|x| \ln 2|N| \\
& \leq \ln \max _{\forall T(x)} \operatorname{Pr}_{G}(x, T(x) \mid \hat{q})+2|x| \ln 2|N| \\
& \leq \ln \operatorname{Pr}_{G}(x \mid \hat{q})+2|x| \ln 2|N|
\end{aligned}
$$

Consequently:

$$
\begin{aligned}
& 0 \leq \ln \operatorname{Pr}_{G}\left(x \mid q^{*}\right)-\ln \max _{\forall T(x)} \operatorname{Pr}_{G}(x, T(x) \mid \hat{q}) \leq 2|x| \ln 2|N|, \\
& 0 \leq \ln \operatorname{Pr}_{G}\left(x \mid q^{*}\right)-\ln \operatorname{Pr}_{G}(x \mid \hat{q}) \leq 2|x| \ln 2|N| .
\end{aligned}
$$

Then, for an ideal estimation algorithm that can compute the global maximum of the two likelihood functions, the difference of the logarithms of the two functions is upper bounded by a linear function of $|x|$ and a logarithmic function of the number of non-terminal symbols. Obviously, this is a very pessimistic bound because we are considering that all parse trees are possible for a given $x$ and a characteristic grammar, without taking into account syntactic restrictions.

Furthermore, we must take into account that both the $\mathrm{IO}$ algorithm and the Viterbi algorithm do not guarantee the achievement of a global maximum. From this fact, an empirical study should be carried out to show the comparative behaviour of the two probabilistic functions and to check how pessimistic the above upper bounds are.

\section{Some Experimental Results}

For the experimental study, two languages were chosen: the palindrome language with three terminal symbols (PAL3) and the arithmetic expression language with 5 terminal symbols (EXP). A SCFG was created for each language and was used only for generating a training sample. These grammars were consistent according to [3]. Each training sample had 5000 strings, but only 630 of them were different for PAL3, and 896 were different for EXP.

For the training process, an initial characteristic grammar was created for each language. The number of non-terminal $(n)$ symbols was chosen heuristically as is described in [10], that is, two times the number of terminal symbols plus 
one ${ }^{1}$ ( 7 for PAL3 and 11 for EXP). Each grammar had the maximum number of rules that could be created with the chosen number of non-terminal symbols and the given number of terminal symbols $(v)$, that is, $n^{3}+n \cdot v{ }^{2}$. The probabilities of the rules were attached randomly, but guaranteeing that the grammar was proper [3]. In order to avoid the problem of a bad initialization, ten different initializations were used for each task and for each algorithm. With this initial grammar, a reestimation process was carried out with the IO algorithm on the one hand and with the Viterbi algorithm on the other hand. At each iteration the values of the two probabilistic functions were calculated for both reestimation algorithms. The number of iterations was determined heuristically by seeing when the function being maximized did not change in two consecutive iterations.

The results obtained in the experimentation for the functions being maximized can be seen in Fig. 2. This figure shows how the two functions evolve through the training process. The difference between the two functions and the theoretical bound for the difference that we deduced in the second section can be seen in Fig. 3. Only two initializations are reported because the behaviour was similar in all cases.

When the IO algorithm was used for training, the approximate likelihood tended to increase in each iteration. In some experiments, it was observed that this value tended to decrease during some iterations. The reason may be that the probability of some rules that were used in the most probable parse tree tended to decrease until those rules were not considered (due to underflow considerations). After this, another parse tree could possibly be in use. Furthermore, it is important to note how the difference between the logarithms of the two functions, that is, the expression (3), tends to decrease. Thus, for example, the theoretical bound for PAL3 was approximately 131,000 (185,500 for EXP). After the first iteration in one initialization, the value of the difference was 57,872 $(105,255)$ and this value decreased down to $2,262(13,349)$, which represents a reduction of $96 \%(87 \%)$ between the first and the last iteration and $98 \%(92 \%)$ between the last iteration and the theoretical bound. Similar comments could be made for the other initialitations.

When the Viterbi algorithm was used for training, it could be observed that the likelihood of the sample tended to increase. In no case did the likelihood of the sample achieve the value of the likelihood when the IO algorithm was used for training. The experiments showed that there was an improvement of the likelihood when using the IO algorithm instead of the Viterbi algorithm. In the ten initializations for PAL3 the improvement was between $55 \%$ and $73 \%$. In the ten initializations for EXP the improvement was between $51 \%$ and $72 \%$. In addition, the difference between the logarithms of the two functions also decreased, but the percentage of the decrease between the first and the last iteration was significantly lower as was observed in any of the tasks. This was

\footnotetext{
${ }^{1}$ Other experiments which are not reported in this work showed that this parameter was not crucial in the comparison of the behaviour of the two functions.

${ }^{2}$ This means that all parse trees are possible and that we were in the worst case as we had supposed with the theoretical bound.
} 

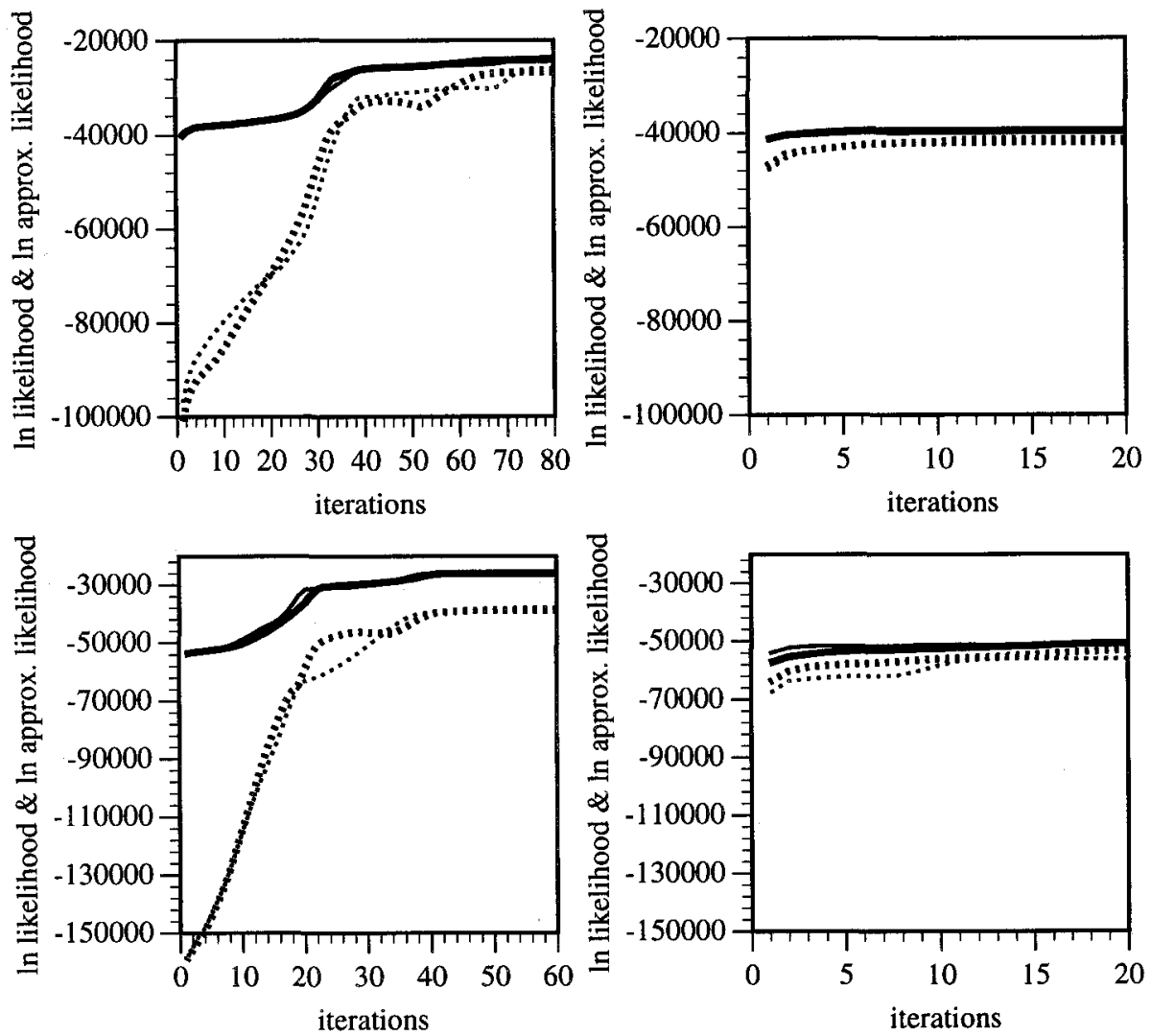

Fig. 2. The results obtained when the IO algorithm (left column) and Viterbi algorithm (right column) were used in the training phase. The continuous lines correspond to the likelihood of the sample and the dashed lines correspond to the approximate likelihood of the sample. The different thicknesses correspond to different initializations. The first row corresponds to PAL3 and the second row to EXP.

due to the syntactic restrictions imposed by the grammar when the Viterbi algorithm was used for training. Nevertheless it is important to note that the real difference between the logarithm of the two functions in the last iteration was $3,275(4,730)$, which represents a reduction of $97 \%(97 \%)$ with respect to the theoretical bound. This value was very similar to the one obtained when the IO algoritm was used for training. This means that the 10 algoritm runs in a way similar to the Viterbi algorithm, that is, the probability of one parse tree increased and the probability of all the other ones decreased. Therefore the mass probability associated to the parse trees without including the best parse tree was very low as also happened when the Viterbi algorithm was used for training. 

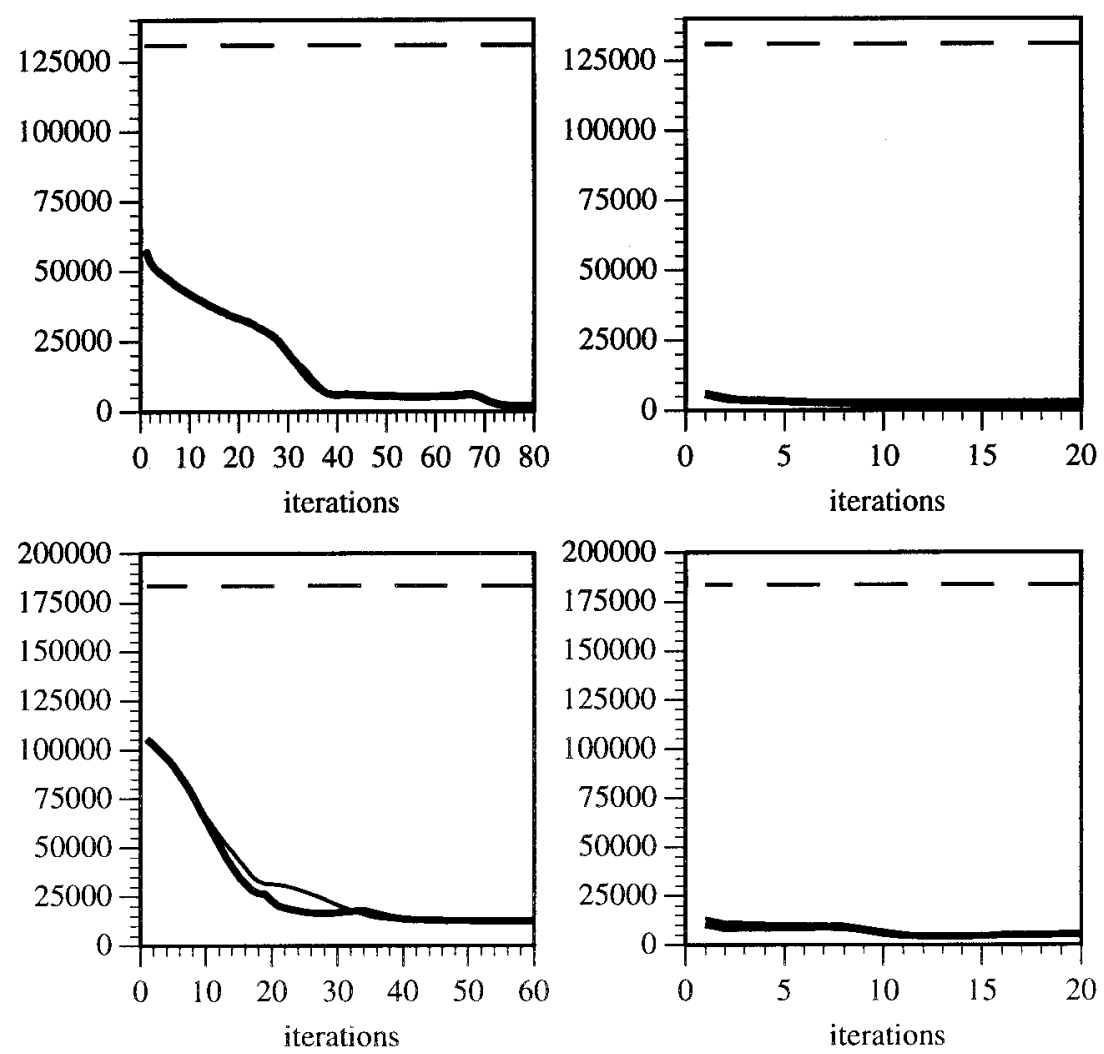

Fig. 3. Difference between the logarithm of the two functions when the IO algorithm (left column) and Viterbi algorithm (right column) were used in the training phase. The different thicknesses correspond to different initializations. The dashed line corresponds to the theoretical bound. The first row corresponds to PAL3 an the second row to EXP.

Also, when the Viterbi algorithm was used for training, it was observed that the likelihood of the sample in some iterations tended to decrease as happened with the approximate likelihood when the IO algorithm was used for training. In this case, the rules that did not participate in the most probable parse tree disappeared and the number of parse trees decreased. This could lead to losses of probability mass which are not compensated by the increase of the probability of the most probable parse tree.

In addition, it is important to note that the approximate likelihood obtained when the IO algorithm was used in the training process for PAL3 was better with the ten initializations than when the Viterbi algorithm was used for training. This also happened in eight out of ten cases for EXP. 


\section{Conclusions}

A theoretical upper bound for the difference between the logarithm of the likelihood of a string and the logarithm of the likelihood of the most probable parse has been shown. For an efficient estimation algorithm that can compute the global maximum of the two likelihood functions, the difference of the logarithms of the two functions is upper bounded by the same bound. This bound is linear with the length of the string and the logarithm of the number of non-terminal symbols, but it is based on very pessimistic assumptions.

An experimental study was carried out to determine the behaviour of the difference between the two functions in a training process. The difference tended to decrease both when the IO algorithm and the Viterbi algorithm were used in the training process. The percentage of the decrease between the first and last iteration was significantly larger when the IO algorithm was used than when the Viterbi algorithm was used. It is important to remark that the difference between the two functions at the end of the training process was very similar for both training algorithms. With regard to the local maxima achieved by both algorithms, in most cases the results obtained by the IO algorithm were better that the ones obtained by the Viterbi algorithm even for the approximate likelihood.

For future work, a better bound could be studied taking into account the syntactic restrictions imposed by the characteristic grammar. If the generative process of SCFG were taken into account, an estimated value to the number of parse trees could be obtained and thus a more realistic bound could be achieved.

\section{Acknowledgment}

The authors thank to the anonymous referees the carefully review of this article.

\section{References}

1. J.K. Baker. Trainable grammars for speech recognition. In Klatt and Wolf, editors, Speech Communications for the 97th Meeting of the Acoustical Society of America, pages 31-35. Acoustical Society of America, June 1979.

2. J.M. Benedí and J.A. Sánchez. Corrective training for the estimation of stochastic context-free grammars. In A. Calvo and R. Medina, editors, Proc. VI Spanish Symposium on Pattern Recognition and Image Analysis, pages 442-450. AERFAI, Abril 1995.

3. T.L. Booth and R.A. Thompson. Applying probability measures to abstract languages. IEEE Transactions on Computers, C-22(5):442-450, May 1973.

4. F. Casacuberta. Grow transformations for probabilistic functions of stochastic grammars. IJPRAI, 10(3), 1996.

5. P. Dupont. Efficient integration of context-free grammars based language models in continuous speech recognition. In New Advances and Trends in Speech Recognition and Coding, pages 179-182. NATO ASI, 1993. 
6. J.E. Hopcroft and J.D. Ullman. Introduction to Automata Theory, Languages and Computation. Addison-Wesley, 1979.

7. E. Horowitz and S. Sahni. Fundamentals of Data Structures in Pascal. Computer Science Press, third edition, 1990.

8. F. Jelinek and J.D. Lafferty. Computation of the probability of initial substring generation by stochastic context-free grammars. Computational Linguistics, $17(3): 315-323,1991$.

9. J. Kupiec. Hidden Markov estimation for unrestricted stochastic context-free grammars. Proc. of ICASSP'92 Vol. 1, pages 177-180. 1992.

10. K. Lari and S.J. Young. The estimation of stochastic context-free grammars using the inside-outside algorithm. Computer, Speech and Language, 4:35-56, 1990.

11. K. Lari and S.J. Young. Applications of stochatic context-free grammars using the inside-outside algorithm. Computer, Speech and Language, (5):237-257, 1991.

12. N. Merhav and Y. Ephraim. Hidden markov modeling using a dominant state sequence with application to speech recognition. Computer Speech and Language, $5: 327-339,1991$.

13. N. Merhav and Y. Ephraim. Maximum likelihood hidden markov modeling using a dominant sequence of states. IEEE. Transactions on Signal Processing, 39(9):2111-2115, 1991.

14. H. Ney. Stochastic grammars and pattern recognition. In P. Laface and R. De Mori, editors, Speech Recognition and Understanding. Recent Advances, pages 319344. Springer- Verlag, 1992.

15. S. Sahni. Concepts in Discrete Mathematics. Camelot Pub. Co., 1985.

16. Y. Sakakibara. Learning context-free grammars from structural data in polynomial time. Theoretical Computer Science, 76:223-242, 1990.

17. Y. Sakakibara, M. Brown, R. Hughey, I.S. Mian, K. Sjölander, R.C. Underwood, and D. Haussle. The application of stochastic context-free grammars to folding, aligning and modeling homologous rna. Computer and Information Science UCSCCRL-94-14, Univ. of California, Santa Cruz, Ca., 1993.

18. A. Stolcke. Bayesian Learning of Probabilistic Language Models. PhD thesis, University of California, Berkeley, CA., 1994.

19. A. Stolcke. An efficient probabilistic context-free parsing algorithm that computes prefix probabilities. Computational Linguistics, 21(2):165-200, 1995. 